\title{
Tuberous sclerosis with negative genetic testing and multiple cerebral cavernomas: A new association (Case report)
}

\author{
ANCA ADRIANA ARBUNE ${ }^{1}$, BASEL ROBERT TORON ${ }^{1}$, IOAN CRISTIAN LUPESCU $^{1}$, \\ IOANA GABRIELA LUPESCU ${ }^{2,3}$, ALIN LAURENTIU TATU ${ }^{4,5}$ and ADRIANA OCTAVIANA DULAMEA ${ }^{1,6}$ \\ ${ }^{1}$ Neurology Department, 'Fundeni' Clinical Institute, 022328 Bucharest; ${ }^{2}$ Department 8-Radiology, \\ Oncology and Hematology, Faculty of Medicine, 'Carol Davila' University of Medicine and Pharmacy, \\ 020021 Bucharest; ${ }^{3}$ Radiology and Medical Imaging Department, 'Fundeni' Clinical Institute, 022328 Bucharest; \\ ${ }^{4}$ Clinical Medical Department, ReForm UDJ, Faculty of Medicine and Pharmacy, 'Dunarea de Jos' University of Galati, \\ 800010 Galati; ${ }^{5}$ Dermatology Department, 'Sf. Parascheva' Clinical Hospital of Infectious Diseases, 800179 Galati; \\ ${ }^{6}$ Department Dental Medicine III, Faculty of Dental Medicine, 'Carol Davila' University of Medicine and Pharmacy, \\ 010237 Bucharest, Romania
}

Received June 18, 2021; Accepted July 19, 2021

DOI: $10.3892 /$ etm.2021.10617

\begin{abstract}
Tuberous sclerosis complex (TSC) is an autosomal dominant disorder with multisystemic involvement usually resulting from mutations in the tuberous sclerosis 1 (TSC1) or TSC2 genes. However, 10 to $25 \%$ of patients do not exhibit these mutations. Cerebral cavernous malformations (CCMs) are capillary-venous malformations that can be asymptomatic or cause variable neurological manifestations, including seizures. Familial CCMs are recognized. In both conditions, specific dermatological lesions are associated. We present the case of a 31-year-old female with TSC diagnosed at the age of 18 years who presented with negative genetic testing. She was admitted to our department in 2019 for a sudden increased frequency of focal seizures. Patient examination revealed multiple facial and intraoral angiofibroma, diplopia, right hemihypoesthesia, brisk deep tendon reflexes, and distal leg paresthesia. VideoEEG indicated a frontal paramedian epileptogenic focus. Cerebral magnetic resonance imaging (MRI) and angioMRI identified multiple fronto-parietal cortical tubers, as well as multiple CCMs, with evidence of bleeding in one. Under antiepileptic drug (AED) and mTOR inhibitor
\end{abstract}

Correspondence to: Dr Anca Adriana Arbune, Neurology Department, 'Fundeni' Clinical Institute, 258 Sos. Fundeni Street, 022328 Bucharest, Romania

E-mail: anca.arbune@gmail.com

Dr Alin Laurentiu Tatu, Clinical Medical Department, ReForm UDJ, Faculty of Medicine and Pharmacy, 'Dunarea de Jos' University of Galati, 35 Alexandru Ioan Cuza Street, 800010 Galati, Romania E-mail: dralin_tatu@yahoo.com

Key words: neuro-cutaneous diseases, tuberous sclerosis with negative genetic testing, multiple cerebral cavernous malformations, seizures, epilepsy, skin lesions treatment, the seizure frequency significantly improved in a short period of time. This is the first reported case of tuberous sclerosis with negative genetic testing associated with multiple cerebral cavernoma. Such complex patients require multidisciplinary management and detailed genetic testing for increasing knowledge on neuro-cutaneous disorders.

\section{Introduction}

Tuberous sclerosis complex (TSC) is an autosomal dominant disorder with multisystemic involvement, usually due to a pathogenic mutation in the tuberous sclerosis 1 (TSCl) or $T S C 2$ genes (1). A positive genetic test is considered sufficient for the diagnosis. However, 10 to $25 \%$ of patients do not exhibit the mentioned mutations upon genetic testing (2). The clinical diagnostic criteria include eleven major features and six minor features from different organ systems and a definite diagnosis requires either two major criteria or one major and at least two minor criteria (2). Infantile spasms or focal seizures usually initially occur during the first two years of life in over $80 \%$ of the patients, with good control of seizures under antiepileptic drugs (AEDs) in more than half of patients (3). The cerebral malformations considered among the diagnostic criteria, also standing at the origin of the epileptic seizures, include subependymal nodules, subependymal giant cell astrocytoma, and cortical dysplasia $(2,3)$.

The dermatological features of TSC make up the largest part of the diagnostic criteria and are present in almost all patients (2). A simple physical examination, with close attention paid to skin lesions, can suggest the diagnosis even soon after birth, making possible the diagnosis of TSC before the appearance of neurological signs or developmental delay (4). This allows swift interventions and treatment. A large trial (EPISTOP) demonstrated that preventive treatment of infants with TSC with vigabatrin reduced the risk and severity of epilepsy (5). The effects of this early intervention have a much larger impact on the chances of these infants to have 
normal development and a long-term quality of life. Previous studies have demonstrated that in children with TSC epilepsy remission is significantly correlated with better cognitive outcomes (6-8).

Cerebral cavernous malformations (CCMs) are capillary-venous malformations with irregular structure found in the brain and in the spinal cord. They can be asymptomatic or can cause variable neurological manifestations, most often seizures $(9,10)$. CCMs usually become evident between the second and fifth decades of life; half of cases are discovered incidentally and around $80 \%$ of cases are sporadic (9). When multiple CCMs are identified or in the event of a single CCM in a person with a positive family history, genetic testing for the pathogenic genes KRIT1/CCM1, MGC4607/CCM2 and $P D C D 10 / C C M 3$ is indicated (10,11). Apart from the neurological features, further supporting evidence for the suspicion of familial CCM are vascular ocular and dermatological lesions, which have been described in different families $(11,12)$.

\section{Case report}

We present the case of a 31-year-old female, diagnosed with TSC from the age of 18, after a first generalized seizure. She was admitted to our clinic in 2019 for increased seizure frequency. Personal history included chronic kidney disease and right nephrectomy due to renal angiomyolipoma. Treatment on admission consisted of lamotrigine $250 \mathrm{mg}$ /day and everolimus $7.5 \mathrm{mg} /$ day. Everolimus treatment was started in 2018 with favorable effects on renal function and a reduction in seizure frequency. Genetic testing for TSC1 and TSC2 mutations was negative. The patient provided informed consent for publication and data usage concerning the case report.

The patient described two types of seizures: i) seizures with motor automatisms of the right leg continuing with bilateral leg clonias, without loss of awareness and duration of up to $1 \mathrm{~min}$; and ii) focal to bilateral tonic-clonic seizures, observed by the family during the night, and without any other details available from the patient, with a duration of up to 2-3 min and postictal sleepiness and confusion. On admission, the patient reported 2-3 focal seizures/day from a previous frequency of 2-4 seizures/month.

Neurological examination revealed an oriented patient, with normal speech, no signs of neck stiffness, diplopia and horizontal nystagmus on right lateral gaze, hemihypoesthesia of right face and limbs, no motor deficits or ataxia, brisk deep tendon reflexes and distal legs paresthesia. General examination showed an overweight patient with no fever, multiple facial and gingival angiofibroma, right latero-lumbar scar post nephrectomy with normal cardiovascular and respiratory functions. We also noted the presence of renal angiomyolipomas, as documented on abdominal ultrasound.

VideoEEG identified frequent epileptiform discharges and slowing of the background rhythm predominantly over the frontal paramedian derivations, with slightly higher amplitudes and spread in the right hemisphere.

Cerebral magnetic resonance imaging (MRI) and angioMRI were performed, revealing numerous hyperintense cortico-subcortical lesions fronto-parietal bilaterally, suggestive of cortical tubers. Additionally, round-oval shaped hypointense lesions were visible on susceptibility weighted imaging (SWI) sequences, located right frontal parasagittal and left juxtaventricular, which were suggestive of cavernomas with hemosiderin deposition (Fig. 1).

The patient was administered lamotrigine $400 \mathrm{mg} /$ day and everolimus $7.5 \mathrm{mg}$ /day and experienced a marked improvement in seizure frequency (1/week). Genetic testing for familial cavernomatosis was proposed, but, unfortunately, she refused this testing due to costs. The patient expressed an intent to have children in the upcoming years, therefore the lamotrigine dose was gradually reduced to $200 \mathrm{mg} /$ day over the next 6 months.

\section{Discussion}

After performing a thorough literature search and to the best of our knowledge, we conclude that this is the first reported case with an association between TSC with negative genetic testing and multiple CCMs in a patient with epileptic seizures.

Most TSC patients have mutations in the TSC1 or TSC2 genes. In a review by Henske et al, multiple studies suggest that the majority of patients have a TSC 2 mutation. The mutations result in a loss of inhibition of the mTOR pathway and consequent overexpression of the mTOR complex, with dysregulation of normal cell functioning (1). However, a significant percentage of patients with clinically diagnosed TSC do not exhibit these mutations, as is the case of our patient, and are thought to have mosaicism or another, yet unidentified, pathomechanism $(1,2)$. Further studies are needed to complete the list of pathogenic mutations which result in TSC, as well as to establish the risk of transmission to the next generation, especially in cases similar to our patient.

One particularity of our case consisted of the late diagnosis of TSC, at the age of 18 years, after a generalized epileptic seizure. After performing a more thorough history, the patient reported a few episodes that suggested epileptic auras (13) or short focal seizures during childhood, which were ignored at that moment and considered isolated events, not requiring medical attention. Previous studies have highlighted the difficulties in the recognition of subtle focal seizures, but commonly, a diagnosis is established within the first years of life $(3,13-15)$.

The main dermatological criteria for TSC diagnosis include: hypomelanotic macules, angiofibromas, fibrous cephalic plaque, shagreen patch, 'confetti' skin lesions, intraoral and ungual fibromas (16). These must be carefully differentiated from similar skin lesions, which can be found in other, associated or independent dermatological conditions, such as vitiligo, lichen sclerosus, lichen planus, single or multiple cutaneous tumors, and localized scleroderma (17-19). At least one type of skin lesions is found in more than $90 \%$ of TSC patients, irrespective of age, the most commonly observed after birth being hypomelanotic macules (20). Our patient exhibited multiple facial and intraoral angiofibromas, which usually appear between ages 2 and 5 years, but in rare cases can also appear later in life, during adulthood (2). The lack of recognition of these typical lesions for TSC until adulthood in our patient underlines the importance of education among general medical practitioners and neurologists on rare inherited neuro-cutaneous disorders, or at least a dermatology referral when suspicious lesions are observed. 
A

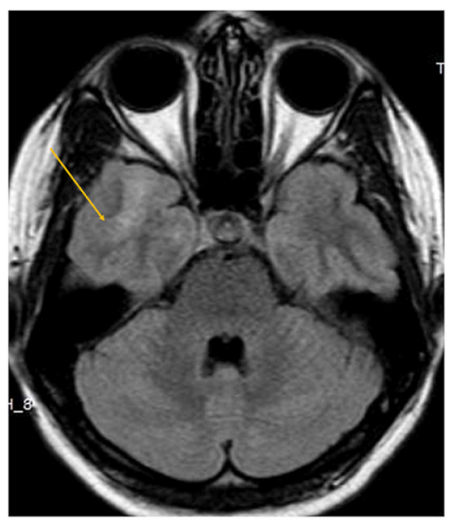

D

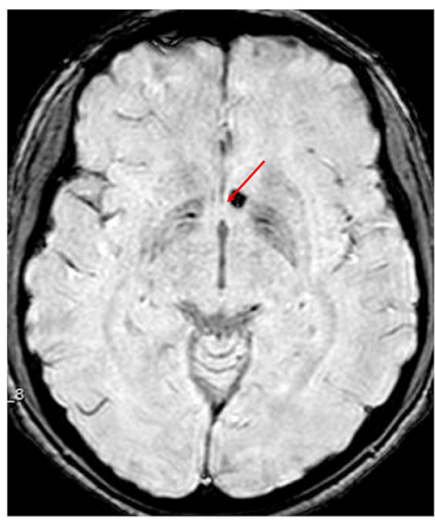

B

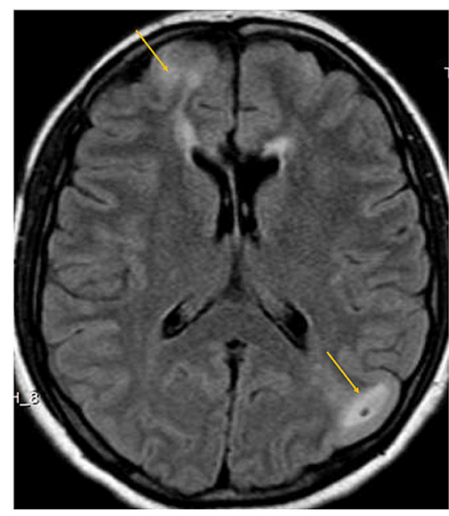

E

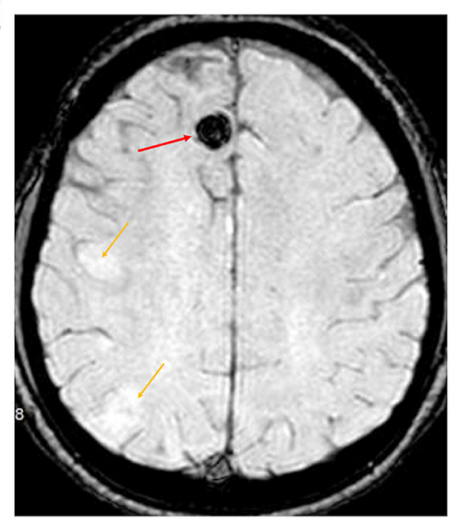

C

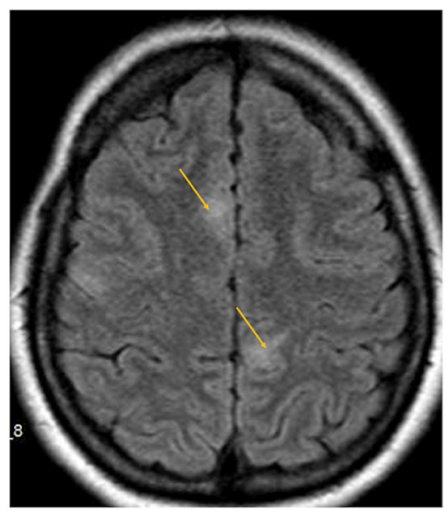

F

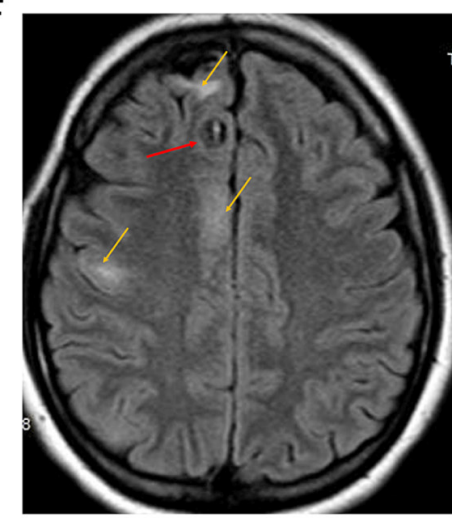

Figure 1. Brain magnetic resonance imaging (MRI) of the 31-year-old female patient with seizures. Orange arrows indicate multiple cortical tubers observed in FLAIR sequence (A-C); red arrows indicate the biggest observed cavernomas, with hemosiderin deposition in the susceptibility weighted imaging (SWI) sequence (D and E), as well as some cortical tubers marked by orange arrows (E); both cortical tubers and a cavernoma in FLAIR sequence are indicated by orange and red arrows, respectively $(\mathrm{F})$.

Another particularity of the case is the presence of multiple CCMs in addition to the typical cortical tubers of TSC. CCMs usually occur as single lesions, predominantly in the brain white matter and the spinal cord. In patients with multiple lesions, approximately $70 \%$ have a familial form $(9,11)$, when one of the pathogenic variants of the genes KRIT1, CCM2 or $P D C D 10$ is inherited. There is a recognized higher risk of bleeding, with the possible emergence of seizures, when multiple CCMs are present $(21,22)$, patient age is younger than 45 years and the presence of a developmental venous anomaly (23).

In cavernoma-related epilepsy, hemosiderin deposits adjacent to cortical tissue and repeated microhemorrhages are thought to induce hyperexcitability due to free radicals and lipid peroxides generated by iron ions $(9,24)$. Considering the clinical data and investigations, we hypothesize that the increase in seizure frequency was secondary to a bleeding in the frontal paramedian cavernoma, which resolved in a short time and required only AED dose adjustments.

Periodical neurological consults in the long run were recommended, especially in the event of a pregnancy, considering a higher risk of recurrent bleeding, as well as a possible increase in seizure frequency, secondary to hemodynamic and hormonal changes related to pregnancy $(3,6,9,21,22,25)$.

Familial CCMs have been described to be associated with a wide array of skin lesions: hyperkeratotic cutaneous capillary-venous malformations (HCCVM), angiokeratoma, cherry angiomas, cutaneous bluish nodules, multiple small bluish papules, capillary vascular anomalies, nodular venous malformations, and port-wine stains $(11,12,26)$. The emergence of these lesions varies from birth to late adulthood $(11,12,26)$. The exact prevalence of dermatological vascular lesions associated with familial CCM is unknown, but one study indicated that 6 of 13 members of an affected family had peculiar skin lesions (11). Even though our patient seemingly had only facial and intraoral angiofibromas, it is possible that some of the facial lesions were actually angiokeratoma, which is associated with a few cases of familial CCM $(27,28)$. Moreover, considering the variable age of the emergence of the dermatological lesions, periodic dermatological consultations were recommended to our patient.

In conclusion, this is the first reported association of tuberous sclerosis with negative genetic testing and multiple cerebral cavernomas in a patient with epilepsy. The collaboration between medical specialists, especially in the fields of neurology and dermatology, is vital in neuro-cutaneous diseases, as certain features relevant to a correct diagnosis may pass unnoticed and may result in a delay in treatment of the affection. On the long term, periodical consultations of both types of specialists are recommended, considering that the emergence of specific lesions or subtle neurological signs can also occur later in life. Multidisciplinary management and whole genome sequencing of such complex cases could lead to a better understanding of the intricacies of inherited neuro-cutaneous disorders and improve the quality of life of these patients. 


\section{Acknowledgements}

Not applicable.

\section{Funding}

No funding was received.

\section{Availability of data and materials}

Any further information regarding this case report can be obtained from the corresponding author upon reasonable request.

\section{Authors' contributions}

AAA performed the analysis and interpretation of the and drafted the manuscript. BRT performed the data collection and assisted in the drafting of the manuscript. IGL played a major role in the acquisition of the data. ICL, ALT, AOD performed the revision of the manuscript for content regarding the literature findings and the patient data. All authors have read and agreed to the published version of the manuscript.

\section{Ethics approval and consent to participate}

Patient written consent was obtained. No ethics committee approval was needed as the article uses only readily available data that the patient agreed to be used (anonymously) for publication. No experimental intervention was performed, and the patient was investigated and treated according to the usual hospital standards.

\section{Patient consent for publication}

Written consent was obtained for publication of the case report.

\section{Competing interests}

The authors declare that they have no competing interests.

\section{References}

1. Henske EP, Jóźwiak S, Kingswood JC, Sampson JR and Thiele EA: Tuberous sclerosis complex. Nat Rev Dis Primers 2: $16035,2016$.

2. Northrup H and Krueger DA; International Tuberous Sclerosis Complex Consensus Group: Tuberous sclerosis complex diagnostic criteria update: Recommendations of the 2012 international tuberous sclerosis complex consensus conference. Pediatr Neurol 49: 243-254, 2013.

3. Nabbout R, Belousova E, Benedik MP, Carter T, Cottin V, Curatolo P, Dahlin M, D'Amato L, D'Augères GB, de Vries PJ, et al: Epilepsy in tuberous sclerosis complex: Findings from the TOSCA study. Epilepsia Open 4: 73-84, 2018.

4. Davis PE, Filip-Dhima R, Sideridis G, Peters JM, Au KS, Northrup H, Bebin EM, Wu JY, Krueger D and Sahin M; Tuberous Sclerosis Complex Autism Center of Excellence Research Network: Presentation and diagnosis of tuberous sclerosis complex in infants. Pediatrics 140: e20164040, 2017.

5. De Ridder J, Lavanga M, Verhelle B, Vervisch J, Lemmens K, Kotulska K, Moavero R, Curatolo P, Weschke B, Riney K, et al: Prediction of neurodevelopment in infants with tuberous sclerosis complex using early EEG characteristics. Front Neurol 11: $582891,2020$.
6. Chu-Shore CJ, Major P, Camposano S, Muzykewicz D and Thiele EA: The natural history of epilepsy in tuberous sclerosis complex. Epilepsia 51: 1236-1241, 2010.

7. Curatolo $P$ and Moavero R: Can we change the course of epilepsy in tuberous sclerosis complex? Epilepsia 51: 1330-1331, 2010

8. Curatolo P, Jóźwiak S and Nabbout R; TSC Consensus Meeting for SEGA and Epilepsy Management: Management of epilepsy associated with tuberous sclerosis complex (TSC): Clinical recommendations. Eur J Paediatr Neurol 16: 582-586, 2012.

9. Rosenow F, Alonso-Vanegas MA, Baumgartner C, Blümcke I, Carreño M, Gizewski ER, Hamer HM, Knake S, Kahane P, Lüders H, et al: Cavernoma-related epilepsy: Review and recommendations for management-report of the surgical task force of the ILAE commission on therapeutic strategies. Epilepsia 54: 2025-2035, 2013.

10. Morrison L and Akers A: Cerebral cavernous malformation, familial. In: GeneReviews ${ }^{\circledR}$ [Internet]. Adam MP, Ardinger HH, Pagon RA, Wallace SE, Bean LJH, Mirzaa G and Amemiya A (eds). University of Washington, Seattle, WA, 1993.

11. de Vos IJ, Vreeburg M, Koek GH and van Steensel MA: Review of familial cerebral cavernous malformations and report of seven additional families. Am J Med Genet A 173: 338-351, 2017.

12. Toll A, Parera E, Giménez-Arnau AM, Pou A, Lloreta J, Limaye N, Vikkula M and Pujol RM: Cutaneous venous malformations in familial cerebral cavernomatosis caused by KRIT1 gene mutations. Dermatology 218: 307-313, 2009.

13. Russo A, Arbune A, Bansal L, Mindruta I, Gobbi G and Duchowny M: The localizing value of epileptic auras: Pitfalls in semiology and involved networks. Epileptic Disord 21: 519-528, 2019.

14. Canevini MP, Kotulska-Jozwiak K, Curatolo P, La Briola F, Peron A, Słowińska M, Strzelecka J, Vignoli A and Jóźwiak S: Current concepts on epilepsy management in tuberous sclerosis complex. Am J Med Genet C Semin Med Genet 178: 299-308, 2018.

15. Ebrahimi-Fakhari D, Mann LL, Poryo M, Graf N, Von Kries R, Heinrich B, Ebrahimi-Fakhari D, Flotats-Bastardas M, Gortner L, Zemlin M and Meyer S: Incidence of tuberous sclerosis and age at first diagnosis: New data and emerging trends from a national, prospective surveillance study. Orphanet J Rare Dis 13: 117, 2018.

16. Teng JM, Cowen EW, Wataya-Kaneda M, Gosnell ES, Witman PM, Hebert AA, Mlynarczyk G, Soltani K and Darling TN: Dermatologic and dental aspects of the 2012 international tuberous sclerosis complex consensus statements. JAMA Dermatol 150: 1095-1101, 2014.

17. Mihăilă B, Dinică RM, Tatu AL and Buzia OD: New insights in vitiligo treatments using bioactive compounds from piper nigrum. Exp Ther Med 17: 1039-1044, 2019.

18. Tatu AL and Nwabudike LC: Male genital lichen sclerosus-a permanent therapeutic challenge. J Am Acad Dermatol 79 (Suppl 1): AB185, 2018.

19. Tatu AL and Nwabudike LC: The treatment options of male genital lichen sclerosus et atrophicus: Treatments of genital lichen sclerosus. In: Proceedings of the 14th national congress of urogynecology and the national conference of the Romanian association for the study of pain, Eforie, pp262-264, 2017.

20. Portocarrero LKL, Quental KN, Samorano LP, Oliveira ZNP and Rivitti-Machado MCDM: Tuberous sclerosis complex: Review based on new diagnostic criteria. An Bras Dermatol 93: 323-331, 2018.

21. Josephson CB, Leach JP, Duncan R, Roberts RC, Counsell CE and Al-Shahi Salman R; Scottish Audit of Intracranial Vascular Malformations (SAIVMs) steering committee and collaborators: Seizure risk from cavernous or arteriovenous malformations: Prospective population-based study. Neurology 76: 1548-1554, 2011.

22. Mouchtouris N, Chalouhi N, Chitale A, Starke RM, Tjoumakaris SI, Rosenwasser RH and Jabbour PM: Management of cerebral cavernous malformations: From diagnosis to treatment. ScientificWorld Journal 2015: 808314, 2015.

23. Kashefiolasl S, Bruder M, Brawanski N, Herrmann E, Seifert V, Tritt S and Konczalla J: A benchmark approach to hemorrhage risk management of cavernous malformations. Neurology 90: e856-e863, 2018

24. Moran NF, Fish DR, Kitchen N, Shorvon S, Kendall BE and Stevens JM: Supratentorial cavernous haemangiomas and epilepsy: A review of the literature and case series. J Neurol Neurosurg Psychiatry 66: 561-568, 1999. 
25. Arbune AA, Craiu D, Cuciureanu ID, Iulian C, Pavel LL, Berbece IS, Grigore CA and Dulamea A: Challenges of valproate treatment during pregnancy: Pros and cons. Rev Chim 71: 456-459, 2020

26. Campione E, Diluvio L, Terrinoni A, Di Stefani A, Orlandi A, Chimenti S and Bianchi L: Progressive late-onset of cutaneous angiomatosis as possible sign of cerebral cavernous malformations. Dermatol Online J 19: 2, 2013.

27. Whitworth WR, Hick RW, Nelson KC and Sidhu-Malik NK Cerebral cavernous malformations associated with cutaneous angiokeratomas and hemangiomas. Cutis 96: 329-332, 2015.
28. Errichetti E, Piccirillo A, Ricciuti F and Ricciuti F: An unusual case of multiple angiokeratomas arising on a medium-sized congenital melanocytic nevus. Indian J Dermatol Venereol Leprol 78: 374-375, 2012.

c) (i) $९$ This work is licensed under a Creative Commons cc) Attribution-NonCommercial-NoDerivatives 4.0 International (CC BY-NC-ND 4.0) License. 